\title{
Os desafios da enfermagem reumatológica: uma perspectiva emergente no cuidado
}

\section{The challenges of rheumatologic nursing: a perspective on emerging care \\ Los retos de la enfermería reumatológica: una perspectiva en el cuidado emergente}

\author{
Alessandra Sant'Anna Nunes'; Helena Maria Scherlowski Leal David ${ }^{I I}$
}

\begin{abstract}
RESUMO: As doenças crônicas compõem um conjunto de condições crônicas que em geral estão relacionadas a causas múltiplas e apresentam curso clínico que muda ao longo do tempo, com possíveis períodos de agudização, podendo gerar incapacidades. Requerem intervenções associadas a mudanças de estilo de vida, em um processo de cuidado contínuo que nem sempre leva à cura. $\mathrm{O}$ presente artigo teve por objetivo apresentar considerações acerca dos desafios da enfermagem no cuidado aos pacientes com doenças crônicas na área da reumatologia e descrever as principais estratégias para promover uma atenção mais eficiente para esse grupo. Pode-se concluir que melhorar os cuidados de saúde para os portadores de doenças crônicas, aqui destacadas as reumatológicas, vai exigir implementação de diretrizes mais eficazes e redesenho de processos e sistemas de oferta de ações e serviços.
\end{abstract}

Palavras-Chave: Cuidado emergente; enfermagem; reumatologia; inovações tecnológicas.

\begin{abstract}
Chronic diseases comprise a group of chronic conditions that are usually related to multiple causes and present clinical courses that change over time, with possible exacerbation periods, which can generate disabilities. They require interventions associated with changes in lifestyle, in a process of continuous care that does not always lead to a cure. This paper aims at considering the challenges to nursing in the care of patients with chronic diseases in the field of rheumatology and at describing the main strategies to promote more effective care to that group. We can conclude that improving health care for patients with chronic diseases, such as the rheumatic ones highlighted here, requires both the implementation of more effective policies and the redesigning of processes and supply systems of actions and services.

Keywords: Emerging care; nursing; rheumatology; technological innovations.
\end{abstract}

RESUMEN: Las enfermedades crónicas constituyen un grupo de condiciones crónicas que por lo general están relacionadas con múltiples causas y presentan curso clínico que cambia con el tiempo, con posibles períodos de exacerbación, que pueden generar discapacidades. Requieren intervenciones asociadas a cambios en el estilo de vida, en un proceso de atención continua que ni siempre conduce a una cura. Este trabajo tuvo como objetivo presentar las consideraciones sobre los retos de la enfermería en el cuidado de pacientes con enfermedades crónicas en el campo de la reumatología y describir las principales estrategias para promover una atención más eficaz a ese grupo. Se puede concluir que la mejora de la atención de salud para los pacientes con enfermedades crónicas, aquí destacadas las reumáticas, requerirá la aplicación de políticas más eficaces y rediseño de procesos y sistemas de oferta de acciones y servicios.

Palabras Clave: Atención emergente; enfermería; reumatología; innovaciones tecnológicas.

\section{INTRODUÇÃO}

O presente artigo teve por objetivo apresentar considerações acerca dos desafios da enfermagem no cuidado aos pacientes com doenças crônicas na área da reumatologia e descrever as principais estratégias para promover uma atenção mais eficiente para esse grupo.

As doenças crônicas compõem um conjunto de condições crônicas que em geral estão relacionadas a causas múltiplas, caracterizadas por início gradual, de prognóstico usualmente incerto, com longa ou indefinida duração. Apresentam curso clínico que muda ao longo do tempo, com possíveis períodos de agudização, podendo gerar incapacidades. Requerem intervenções associadas a mudanças de estilo de vida, em um processo de cuidado contínuo que nem sempre leva à cura ${ }^{1}$.

Atualmente, as condições crônicas são responsáveis por $60 \%$ de todo o ônus decorrente de doenças no mundo. O aumento na prevalência desses agravos é tão vertiginoso que, no ano 2020, $80 \%$ da carga de doenças dos países em desenvolvimento devem advir de problemas crônicos. Nesses países, a aderência aos tratamentos chega a ser apenas de 20\%, levando a estatísticas negativas na área da saúde, com encargos muito elevados para a sociedade, o governo e os familiares. Até hoje, em todo

IMestre em Enfermagem pela Universidade do Rio de Janeiro. Professora Assistente do Departamento de Enfermagem Médico-Cirúrgica, Faculdade de Enfermagem, Universidade do Estado do Rio de Janeiro. Brasil. E-mail: asantnunes@gmail.com.

"Doutora em Saúde Coletiva. Professora Adjunta do Departamento de Enfermagem em Saúde Pública e do Programa de Pós-Graduação em Enfermagem,

Faculdade de Enfermagem, Universidade do Estado do Rio de Janeiro. Brasil. E-mail: helena.david@uol.com.br. 
o mundo, os sistemas de saúde não possuem um plano de gerenciamento das condições crônicas; simplesmente tratam os sintomas quando aparecem² ${ }^{2}$.

Ao reconhecer a oportunidade de melhorar os serviços de saúde para as condições crônicas, a Organização Mundial da Saúde (OMS) lançou o projeto Cuidados Inovadores para Condições Crônicas (CICC) - um modelo abrangente para atualizar os serviços de saúde com vistas a tratar as condições crônicas, com um enfoque na aderência a tratamentos de longo prazo.

$\mathrm{O}$ aumento da expectativa de vida e das doenças crônicas no mundo ocidental trouxe uma maior demanda de cuidados de saúde em todos os níveis de atenção. Esta demanda levou a inovações na organização e oferta de ações de saúde, nas quais enfermeiros e outros profissionais começaram a assumir papéis estendidos. Esta abordagem permite maior flexibilidade para a prestação de cuidados, dado que o paciente é considerado o centro do processo de cuidar e a tradicional opinião central do profissional vai se tornando menos importante ${ }^{3}$.

Assim, cada vez mais o cuidado do enfermeiro vem se tornando relevante na efetividade do tratamento de pacientes com doenças crônicas, sempre baseadas em protocolos dirigidos para as necessidades dos mesmos, visando dar suporte aos desafios das inovações nessa área. Esses desafios vão da tomada de decisão clínica ao primeiro contato à reabilitação do paciente.

Baseados na efetividade do tratamento desses pacientes em condições crônicas, os enfermeiros atuam na perspectiva de um cuidado ampliado, assumem o seu próprio paciente, estabelecem e programam intervenções de enfermagem. Este modelo de atenção tem sido utilizado com sucesso no manejo de outras doenças crônicas, nas quais os pacientes necessitam de acompanhamentos regulares e de monitoramento, tais como diabetes, doença obstrutiva crônica e insuficiência cardíaca ${ }^{4}$.

Um programa de manejo de doença crônica e a avaliação da qualidade de vida têm sido propostos, devido à complexidade dessas condições. É premente a necessidade de intervenções que tenham o objetivo de reduzir a progressão da doença, diminuir hospitalizações, mortalidade e custos, atenuando os impactos negativos da doença para o indivíduo, família e sociedade ${ }^{5}$.

Esse tem sido um estilo de atenção que permite a aplicação das medidas inovadoras para um cuidado diferenciado - novo modelo ampliado, aplicado através de estratégias de adesão, manejo, manutenção e monitoramento dos pacientes em condições crônicas.

\section{Perspectivas}

\section{Um novo modelo ampliado: CICC}

O novo modelo ampliado de cuidado representa um passo importante para os responsáveis pela elaboração de políticas, planejamentos de setores de saúde e outros agentes relevantes para o empreendimento de ações que visem à redução de ameaças impostas pelas condições crônicas ${ }^{2}$.

São propostos, pelo novo modelo ampliado, oito elementos essenciais para aprimorar os sistemas de saúde as condições crônicas. São eles: Apoiar uma mudança de paradigma; gerenciar o ambiente político; desenvolver um sistema de saúde integrado; alinhar políticas setoriais para maximizar os resultados da saúde; aproveitar melhor os recursos humanos do setor saúde; centralizar o tratamento no paciente e na família; apoiar os pacientes em suas comunidades; enfatizar a prevenção com estratégias para minimizar o surgimento das condições crônicas e suas complicações ${ }^{2}$.

Cabe ressaltar que além dos elementos essenciais para aprimorar os sistemas de saúde a implantação de uma Rede de Atenção às Pessoas com Doenças Crônicas também é essencial. No entanto, o bom funcionamento das Redes de Atenção à Saúde (RAS) depende primordialmente da existência de um trabalho compartilhado entre os profissionais da atenção básica e os especialistas focais. Por especialistas focais, entendemos não somente os médicos, mas outros profissionais, como os enfermeiros, cuja atuação é sabidamente efetiva e eficiente ${ }^{6}$.

A implantação da Rede de Atenção às Pessoas com Doenças Crônicas tem o objetivo de promover mudanças na atenção à saúde, que são críticas e estão mais ligadas às modificações no processo de trabalho das equipes, que devem acontecer em todos os pontos de cuidado, desde a Atenção Básica à Saúde (ABS), passando pela Atenção Ambulatorial Especializada (AAE), pela urgência e pelo cuidado hospitalar e domiciliar ${ }^{1}$.

O conhecimento profundo sobre a população usuária de um serviço é elemento essencial para a organização da atenção. Isso possibilita romper com a atenção baseada na oferta de consultas e procedimentos desorganizados, característica dos sistemas fragmentados ${ }^{1}$.

Diversas são as doenças crônicas que atingem a população brasileira e o conhecimento da epidemiologia que envolve cada uma, assim como as necessidades de cuidado, é essencial para uma assistência com mais qualidade e eficiência. Nessa perspectiva, este artigo aborda os elementos que afetam o paciente crônico com doença reumatológica.

\section{Desafios da enfermagem reumatológica}

Um recorte histórico se faz necessário para a discussão dos desafios da enfermagem em reumatologia. Por volta de 1980, no Reino Unido, o cuidado de enfermagem em reumatologia começou em Leeds, onde os pacientes que fizeram parte de um ensaio clínico e ao concluir o estudo, foram devolvidos para o ambulatório geral de reumatologia, e começaram a procurar as enfermeiras para acompanhamento de suas consultas?. 
Esse movimento fez com que fossem criadas as clínicas de enfermagem, que funcionavam ao lado das clínicas dos reumatologistas e prestavam cuidados de educação ao paciente, aconselhamento e apoio. Esse modelo de atenção foi replicado por todo o Reino Unido, com a contratação, nos centros de reumatologia, de enfermeiros especialistas para acompanhamento desses pacientes.

Ainda no Reino Unido, foi realizada uma revisão sistemática com o objetivo de determinar a eficácia do tratamento conduzido por enfermeiros em pacientes com artrite reumatoide (AR), isto é, identificar se produziam efeitos que eram semelhantes aos cuidados médicos tradicionais. Para atender a esse objetivo buscaram estudos que comparassem uma determinada intervenção com outra bem sucedida e já estabelecida no cuidado.

Todos os estudos avaliaram a eficácia dos cuidados conduzidos pelos enfermeiros - enfermeiros especialistas ou enfermeiros clínicos - e todos os pacientes recebedores dos cuidados tinham artrite reumatoide com tempo de doença variável e foram assistidos para acompanhamento clínico ou monitoramento do uso de drogas. Esse estudo concluiu que não existem evidências suficientes para definir se existe diferença significativa no cuidado realizado pelo enfermeiro, quando comparado com aquele desenvolvido por outros profissionais, principalmente os profissionais médicos e destaca que o fator limitante é a falta de estudos clínicos randomizados de boa qualidade para fornecer evidências da eficácia do cuidado de enfermeiros em pacientes com artrite reumatoide ${ }^{8}$.

Para tecer algumas considerações acerca do papel do enfermeiro no manejo das doenças reumatológicas, toma-se como exemplo a AR, condição na qual mais de $90 \%$ dos pacientes sofrem de sintomas em suas articulações da mão e que podem ser seriamente incapacitantes na execução das atividades diárias, de trabalho ou de lazer.

Uma gestão integral tem sido defendida e utilizada para lidar com as consequências da AR. Os pacientes devem ser envolvidos como parceiros de seus cuidados e as dimensões físicas e emocionais de sua doença devem ser abordadas e reconhecidas por eles.

Uma pesquisa holandesa baseada no atendimento multidisciplinar para pacientes com AR demonstrou que um enfermeiro especialista pode ser um elemento importante para uma equipe multidisciplinar. Além disso, o estudo apresentou diversos outros pontos que corroboram essa assertiva, entre os quais se destacam dois.

$\mathrm{O}$ primeiro ponto refere-se ao que outros estudos já apontam: o cuidado do enfermeiro é eficaz, seguro e leva a um maior controle dos sintomas e maior autocuidado do paciente; o cuidado prestado por enfermeiros, com base em protocolos, aumenta a satisfação do paciente e é o tratamento preferido, dentro de uma perspectiva de saúde econômica; formas inovadoras de prática avançada de enfermagem podem aumentar a eficácia do processo de cuidado quando são incorporadas à uma estrutura de trabalho que é internamente consistente, ajustada para o ambiente, e que disponibiliza diversas habilidades ${ }^{9}$.

E um segundo aspecto do estudo versa sobre a implicação para a prática clínica e política, e afirma que o enfermeiro incluído em consulta multidisciplinar deve focar especialmente nos pacientes que apresentam necessidades que demandam aconselhamento extenso, sendo que a redução de custos não deve ser esperada diretamente após a inclusão de um enfermeiro, já que a realização de novas ações de saúde, por profissionais novos, representam, num primeiro momento, custos adicionais para os sistemas de saúde 9 .

No entanto, os estudos permitem afirmar que apesar de não existir, inicialmente, uma redução de custos o cuidado prestado pelo enfermeiro especialista é eficaz e deve ser incorporado à estrutura do novo modelo de atenção à saúde na área da reumatologia e em todas as demais que envolvem o cuidado ao paciente crônico.

\section{Conhecimentos básicos da reumatologia na atenção ambulatorial especializada}

O enfermeiro especialista em reumatologia deve ser bem informado sobre os processos de doença e princípios da prática pertinente à especialidade de reumatologia, ser capaz de identificar e gerenciar diversos problemas que os pacientes com doenças reumáticas podem desenvolver, tais como: manejo da dor, alteração no conforto com o surgimento da rigidez, alteração no nível de energia com a fadiga, mudanças na mobilidade física, distúrbio de autoconceito, decisões de autogestão, diversidades etnoculturais da percepção da doença por pacientes e familiares, autocuidado, participar na política de saúde, no planejamento e desenvolvimento das ações ${ }^{10}$.

A avaliação do paciente e o plano de tratamento são de suma importância para o sucesso no cuidado de enfermagem em reumatologia. Um resumo dos pontos principais que envolvem a avaliação e o tratamento do paciente, propostos pela Associação das Enfermeiras Americanas e pelos Profissionais de Saúde da Associação de Artrite, é apresentado na Figura $1^{11}$.

Além da avaliação criteriosa e o plano de tratamento especializado, um dos papéis fundamentais do enfermeiro especialista em reumatologia é promover a adesão ao tratamento e compreensão dos riscos e benefícios da terapia medicamentosa, encorajando o seu uso efetivo e confiável ${ }^{12}$.

A falta de adesão ao tratamento tem se tornado um problema básico que afeta muitos pacientes com doenças crônicas. Apesar disso, ainda há poucos estudos que abordam essa temática. Até o momento, poucas técnicas têm se mostrado efetivas para aumentar a adesão ao tratamento, tornando esse desafio cada vez maior para os enfermeiros especialistas. Em síntese, os estudos de metanálise e revisão sistemática que avaliam intervenções para o aumento da adesão 


\begin{tabular}{|c|c|}
\hline AVALIAÇÃO CLÍNICA DO PACIENTE & PLANO DE TRATAMENTO \\
\hline $\begin{array}{l}\text { Reconhecer os sintomas e incapacidades funcionais } \\
\text { associados aos diversos processos de doença } \\
\text { reumatológica. }\end{array}$ & $\begin{array}{l}\text { Desenvolver estratégias de gestão apropriadas para } \\
\text { lidar com processos específicos de doença do } \\
\text { paciente. }\end{array}$ \\
\hline $\begin{array}{l}\text { Diferenciar os sinais de aguda contra a dor crônica, } \\
\text { rigidez e fadiga. }\end{array}$ & $\begin{array}{l}\text { Demonstrar um entendimento das modalidades } \\
\text { farmacológicas e não farmacológicas adequadas } \\
\text { para tratamento de problemas do paciente. }\end{array}$ \\
\hline $\begin{array}{l}\text { Identificar fatores como o sono, energia, mobilidade, } \\
\text { postura, alimentação, relações interpessoais, e saúde } \\
\text { geral, que pode contribuir para a percepção da dor, } \\
\text { fadiga, rigidez e outros sintomas associados à doença } \\
\text { reumática. }\end{array}$ & $\begin{array}{l}\text { Compreender e avaliar os parâmetros laboratoriais } \\
\text { necessários para monitorar a progressão da doença } \\
\text { e fornecendo orientação na gestão farmacológica. }\end{array}$ \\
\hline $\begin{array}{l}\text { Avaliar o regime medicamentoso em relação aos } \\
\text { efeitos colaterais, reações adversas, e eficácia. }\end{array}$ & $\begin{array}{l}\text { Utilizar escalas de avaliação funcional } \\
\text { apropriados. }\end{array}$ \\
\hline $\begin{array}{l}\text { Detectar mudanças sutis no comportamento do } \\
\text { paciente que podem sugerir alteração na doença. }\end{array}$ & $\begin{array}{l}\text { Demonstrar compreensão dos fatores psicológicos } \\
\text { que podem estar relacionados a estados de } \\
\text { doenças crônicas e do processo de ajuste posterior, } \\
\text { (incluindo a imagem do corpo, a percepção da } \\
\text { dor, e depressão). }\end{array}$ \\
\hline $\begin{array}{l}\text { Identificar intervenções preventivas ou terapêuticas } \\
\text { úteis na resolução dos problemas do paciente. }\end{array}$ & $\begin{array}{l}\text { Avaliar a capacidade e motivação para om } \\
\text { paciente participar do seu plano de cuidados. }\end{array}$ \\
\hline $\begin{array}{l}\text { Realizar um exame físico adequado para avaliar o } \\
\text { problema do paciente e reconhecer sinais associados } \\
\text { com vários processos de doenças reumáticas. }\end{array}$ & $\begin{array}{l}\text { Selecionar modalidades de ensino apropriadas } \\
\text { para paciente e / ou sistema de apoio. }\end{array}$ \\
\hline $\begin{array}{l}\text { Reconhecer os indicadores físicos e psicológicos } \\
\text { sugestivos de processo da doença. }\end{array}$ & $\begin{array}{l}\text { Demonstrar sensibilidade para a importância da } \\
\text { dinâmica familiar, estilos de comunicação, e as } \\
\text { diferenças culturais que podem afetar pacientes } \\
\text { que lidam com uma doença crônica. } \\
\text { Identificar as medidas preventivas e terapêuticas } \\
\text { adequadas necessárias para preservar e / ou } \\
\text { melhorar a função. }\end{array}$ \\
\hline
\end{tabular}

FONTE: Padrões de resultados para a prática de enfermagem reumatologia ${ }^{12}$.

FIGURA 1: Avaliação e Tratamento do paciente com doença reumatológica propostos pela Associação das Enfermeiras Americanas.

ao tratamento concluem que não existe apenas uma estratégia mas uma soma de estratégias, a cargo de diversos especialistas, que podem contribuir efetivamente para superar tala problema ${ }^{13}$.

Entre as estratégias citadas por diversos autores, ressaltam-se a educação e o apoio de pacientes com doenças crônicas, como as reumatológicas. Fica claro que enfermeiros especialistas em reumatologia têm um papel importante na educação e no apoio de pacientes com $\mathrm{AR}^{14}$. No entanto, ainda falta conhecimento da própria perspectiva de educação que se devemos proporcionar aos pacientes reumatológicos.

Em um estudo com terapia medicamentosa que avaliou a satisfação dos pacientes sobre as informações que foram fornecidas, pelos enfermeiros, sobre o uso de drogas antirreumáticas prescritas a eles, cerca de metade não estava satisfeita com as informações dadas. A principal razão para insatisfação, com o programa educacional, foi que o enfermeiro não se concentrou no bem-estar emocional dos pacientes com AR e sim em questões relacionadas ao tratamento ${ }^{15}$.

Os resultados forneceram uma visão útil para a educação do paciente com doença reumatológica, ao destacarem que os enfermeiros devem evitar apenas a transmissão de informações de uma forma rotineira e sim favorecer a discussão dos sentimentos de seus pacientes e preocupações, especialmente com pacientes recém-diagnosticados. A educação do paciente deve equilibrar as informações que os pacientes precisam para aderir ao tratamento e conhecimento sobre sua patologia, com a sua necessidade de apoio emociona $1^{15}$.

Estudos apontam que o conhecimento dos pacientes e de seus cuidadores sobre a sua doença é rudimentar e limitado às sequelas apresentadas pelos pacientes, observando-se um despreparo dos cuidadores. Salienta-se, mais uma vez, a importância da educação em saúde junto a pacientes e familiares/cuidadores sobre os temas - a doença, reconhecimento dos sinais e sintomas, controle dos fatores de risco e cuidados domiciliares após a alta hospitalar ${ }^{16}$.

Objetivando atingir o equilíbrio esperado para o tratamento, enfermeiros da clínica de reumatologia do Hospital de Doenças Comuns da Universidade de Nova Iorque perceberam a necessidade de oferecer aos pacientes mais informações sobre sua condição de saúde do que foi, rotineiramente, dada durante as 
visitas clínicas que os mesmos receberam. O grande objetivo do projeto desenvolvido por esses profissionais foi de apoiar o envolvimento do paciente na decisão terapêutica e incentivar a sua participação no planejamento do tratamento. Para tal, a equipe de enfermeiros desenvolveu um programa educacional com base em vários tópicos relacionados à artrite, como: uso de medicamentos, controle da dor, nutrição, saúde sexual, estresse, terapia física e problemas psicossociais ${ }^{14}$.

Destaca-se, portanto, a dimensão educativa, constituinte da prática do enfermeiro, como um elemento central para a sua atuação em reumatologia. Sem prescindir do conhecimento clínico, trata-se de proporcionar ao paciente portador de um agravo limitante e sem perspectiva de cura, apenas de controle, não somente um suporte cognitivo que mantenha e estimule a autonomia no autocuidado diário, inclusive o medicamentoso ${ }^{17}$, mas também que se ofereça como espaço de interação e acolhimento às demandas emocionais, reafirmando o caráter integral do cuidado de enfermagem.

\section{ConClusão}

Pode-se concluir que melhorar os cuidados de saúde para os portadores de doenças crônicas, aqui destacadas as reumatológicas, vai exigir implementação de diretrizes mais eficazes e redesenho de processos e sistemas de oferta de ações e serviços. $\mathrm{O}$ encaminhamento dos pacientes para atendimento especializado é um componente chave da função do sistema de saúde, e que sabemos que ainda precisa ser aperfeiçoado no sistema público brasileiro. Os cuidados destinados aos pacientes com doenças crônicas de média e alta complexidade, que são atendidos na atenção ambulatorial especializada, ainda precisam ser mais estudados e ampliados. A eficácia do tratamento para problemas de saúde complexos pode ser maior quando são incluidos as diretrizes para o cuidado, o papel do provedor, a codificação uniforme das ações e serviços, com destaque para a educação do paciente, o que prevê pré-consulta de gerenciamento de referência da especialidade e monitoramento do processo de gestão dos cuidados.

Por fim o tratamento das doenças reumáticas se torna mais eficiente quando realizado por uma equipe multidisciplinar, com um acompanhamento regular do paciente, envolvendo adesão à terapia medicamentosa, exercício físico leve a moderado, fisioterapia, proteção articular, autogestão do cuidado(inclusive o medicamentoso), educação em saúde e de apoio psicossocial. Este fator, portanto, convoca a enfermagem a assumir mais esse desafio, com vistas ao melhor cuidado possível à saúde das pessoas, nos níveis tanto individual como coletivo.

\section{REFERÊNCIAS}

1.Ministério da Saúde $(\mathrm{Br})$. Secretaria de Atenção à Saúde. Departamento de Atenção Básica. Diretrizes para o cuidado das pessoas com doenças crônicas nas redes de atenção à saúde e nas linhas de cuidado prioritárias. Brasília (DF): Ministério da Saúde; 2013.

2.Organização Mundial da Saúde (OMS) . Cuidados inovadores para condições crônicas: componentes estruturais de ação: relatório mundial. Brasília (DF): OMS; 2003. 3.Richardson G, Maynard A, Cullum N, Kindig D. Mudanças: substituição ou desenvolvimento de serviços? Política de Saúde. Brasília (DF): Ministério da Saúde; 1998.

4.Carey N, Courtenay M. A review of the activity and effects of nurse-led care in diabetes. Journal of Clinical Nursing. 2007; 16: 296-304.

5.Goulart FAA, Kemper ES. Doenças crônicas não transmissíveis: estratégias de controle e desafios e para os sistemas de saúde. Brasília (DF): Ministério da Saúde; 2011. 6.Mendes EV. O cuidado das condições crônicas na Atenção Primária à Saúde: o imperativo da consolidação da Estratégia Saúde da Família. Brasília (DF): Organização Pan-Americana da Saúde; 2012.

7.Phelan MJI, Byrne J, Campbell A, Lynch MP. A pro[le of the rheumatology nurse specialist in the United Kingdom. Rheumatology. 1992; 31: 858-9.

8.Ndosi M, Vinall K, Hale C, Bird H, Hill J. The effective ness of nurse-led care in people with rheumatoid arthritis: a systematic review. Int J Nurs Stud. 2011; 48: 642-54. 9.Van Der Sluis CK, Datema L, Saan I, Stant D, Dijkstra PU. Effects of a nurse practitioner on a multidisciplinary consultation team. Journal of Advanced Nursing. 2009; 65: 625-33. 10.Pigg JS. Rheumatology in nursing: an evolution of role and functions of a small speciality. Arthritis Treatment. 1990; 3:109-15.

11.American Nurses Association. Padrões de resultados para a prática de enfermagem reumatologia. 1983; I-II:1-10. 12.Homer D, Nightingale P, Jobanputra P. Providing patients with information about disease-modifying anti-rheumatic drugs: individually or in groups? A pilot randomized controlled trial comparing adherence and satisfaction. Musculoskeletal care. 2009;7:78-92.

13.Castro MS, Fuchs FD. Abordagens para aumentar a adesão ao tratamento em pacientes com hipertensão arterial de difícil controle. Rev Bras Hipertensão. 2008; 15(1):25-7. 14.Lemon J, Marichal-Williams B, Blum E, Bracero M. A Nurse-Led Project Orthopaedic Nursing. Rheumatoid Arthritis Educational Series. 2012; 31:4-12.

15.Makelainen P, Vehvila KJ, Anna MP. Rheumatoid arthritis patient education: RA patients experience. Journal of Clinical Nursing. 2009; 18:2058-65.

16.Souza NPG, Maniva SJCF, Freitas CHA. O conhecimento de acompanhantes/cuidadores de vitimados por acidente vascular cerebral no contexto hospitalar. Rev enferm UERJ. 2013; 21:101-5.

17.Souza RFF, Silva LD. Estudo exploratório das iniciativas acerca da segurança do paciente em hospitais do Rio de Janeiro. Rev enferm UERJ. 2014; 22:22-8. 